\title{
Restoration of RI-beams from a projectile fragment separator by Laser Ionization gas Catcher -PALIS-
}

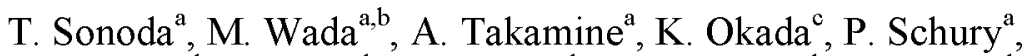

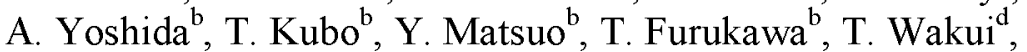 \\ T. Shinozuka ${ }^{d}, H$. Iimura ${ }^{e}, Y$. Yamazaki ${ }^{a}$, I. Katayama ${ }^{f}$, \\ S. Ohtani ${ }^{\mathrm{g}}$, H. Wollnik ${ }^{\mathrm{h}}$, H.A. Schuessler ${ }^{\mathrm{i}}$, Yu. Kudryavtsev ${ }^{\mathrm{j}}$, \\ P. Van Duppen ${ }^{\mathrm{j}}, \mathrm{M}$. Huyse $\mathrm{e}^{\mathrm{j}}$, and the SLOWRI Collaboration \\ ${ }^{a}$ Atomic Physics Laboratory, RIKEN, 2-1 Hirosawa, Wako, Saitama 351-0198, Japan \\ ${ }^{b}$ Nishina Center for Accelerator Based Science, RIKEN, 2-1 Hirosawa, Wako, Saitame 351-0198, Japan \\ ${ }^{c}$ Department of Physics, Sophia University, 7-1 Kioicho, Chiyoda, Tokyo 102-8554, Japan \\ ${ }^{d}$ Cyclotron and Radioisotope Center, Tohoku University, Sendai 980-0191, Japan \\ 'Japan Atomic Energy Agency (JAEA), Tokai, Ibaraki 319-1184, Japan \\ Institute of Particle and Nuclear Studies, KEK, Tsukuba, Ibaraki 305-0801, Japan \\ ${ }^{g}$ Institute for Laser Science, University of Electro-Communications, Chofu, Tokyo 182-8585, Japan \\ ${ }^{h}$ II. Physikalisches Institute, Justus-Liebig-Universität Giessen, Giessen, Germany \\ ${ }^{i}$ Department of Physics, Texas A\&M Universirty, College Station, TX 77843, USA \\ ${ }^{j}$ Instituut voor Kern- en Stralingsfysica, K. U. Leuven, Celestijnenlaan 200D, B-3001, Lewven, Belgium
}

\begin{abstract}
A fragment separator at heavy ion accelerator facilities is a versatile instrument to provide wide variety of radioactive isotope (RI) beams. However, more than $99.99 \%$ of precious RI-ions are simply dumped in the slits or elsewhere in the fragment separator. A novel concept to restore such RI-ions for parasitic slow RI-beams is proposed. Installation of a laser ionization gas catcher in the vicinity of the first or second focal point of the fragment separator enables to collect dead isotopes in the slits. The design concept and expected performance are discussed.
\end{abstract}

Keywords: Fragment separator, Gas catcher, Laser ion source, Resonance ionization.

PACS: $29.25 . \mathrm{Ni}, 29.25 . \mathrm{Rm}, 32.80 . \mathrm{Fb}, 41.85 . \mathrm{Ar}, 29.38$.-c

\section{INTRODUCTION}

A new heavy ion accelerator facility has been constructed at RIKEN and is now in the commissioning stage. The Radioactive Ion Beam Factory (RIBF) consists of a linear accelerator, four cyclotrons and a projectile fragment separator, BigRIPS [1], which provides a wide variety of short-lived radioactive isotope (RI) ions without restrictions on their lifetimes or chemical properties. The RI-beams are mainly used for secondary nuclear reactions at high energy $(>100 \mathrm{~A} \mathrm{MeV})$. The beams are, however, not adequate for low energy beam experiments, in particular for trapping experiments in Penning traps or in similar devices that allow a variety of high precision experiments. We therefore proposed a universal slow RI-beam (SLOWRI) facility as a principal facility of RIBF using a gas catcher cell with an rf-carpet ion guide [2]. At SLOWRI, the relativistic RI-beams are decelerated and cooled in He gas cell and the 
thermal ions are efficiently collected by inhomogeneous of field and extracted as low energy $(<\mathrm{eV}) \mathrm{RI}$-beams for a wide range of precision atomic spectroscopy. Since there are no chemical processes in production, separation, deceleration and cooling, the facility, in principle, can provide isotopes of all elements. The research \& development work for SLOWRI is successfully progressing and some spectroscopy experiments on Be ions have been performed [3,4]. Beam time at such a modern accelerator facility is, however, always limited and operational costs are often high. We therefore propose an additional new method to drastically enhance the usability of such an expensive facility by employing a parasitic production of slow RI-beams. At RIBF, a single primary beam produces thousands of isotopes simultaneously but only a few isotopes are used for an experiment while the other $>99.99 \%$ of isotopes are simply thrown away. Figure 1 shows one example of the yield map of a projectile fragmentation from a $350 \mathrm{MeV} /$ nucleon ${ }^{136} \mathrm{Xe}$ beam of $0.5 \mathrm{p} \mu \mathrm{A}$. Many nuclides are simultaneously produced from a single ion beam. If ${ }^{78} \mathrm{Ni}$ beam is selected through the fragment separator, many other nuclides are freely available at the first focal point (F1) or the second focal point (F2) in the fragment separator.

We propose a novel method to collect such dead isotopes for slow RI-beams by installing laser ionization gas catchers in the vicinity of the first or second focal point of the fragment separator BigRIPS, which will provide parasitic RI-beams with free of extra operational cost. When necessary, multiple gas cells can be located at several places in the BigRIPS and multiple RI-beams can be simultaneously extracted.

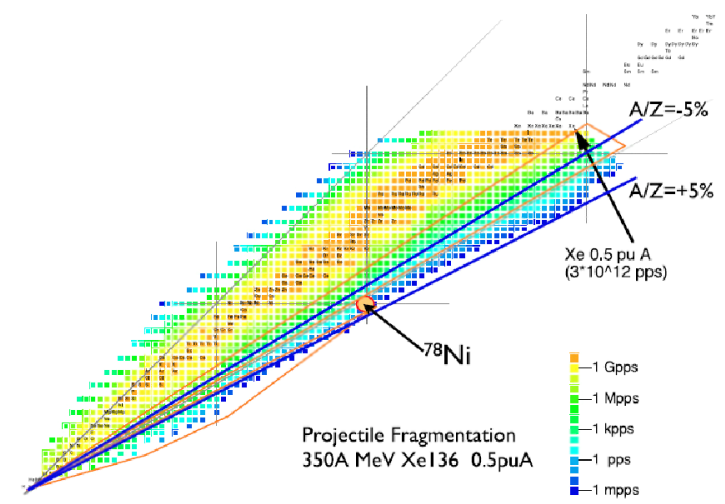

FIGURE 1. Nuclides simultaneously produced from a single primary beam of ${ }^{136} \mathrm{Xe}$. Many nuclides having $+-5 \%$ different $A / Z$ from the main beam $\left({ }^{78} \mathrm{Ni}\right.$, in this example) are presented in areas $+-10 \mathrm{~cm}$ apart from the main beam at the first focal point.

\section{DESIGN OF THE PROPOSED LASER IONIZATION GAS CATCHER}

The fragment separator, BigRIPS, consists of two set of magnetic separators with an energy degrader in between. The first separates the beams by the mass to charge ratio, $A Z$, while the second separates by $\approx A^{2.5} / Z^{1.5}$ due to the energy degrader after the first separator. In this way, a single isotope beam is, in principle, obtained by the two- 
stage separation. All contaminant ions with slightly different $A / Z$ are stopped in the slit at $\mathrm{F} 1$ and dozens of isotopes in the vicinity of the desired isotope are stopped in the slit at F2. Our aim is to collect those isotopes lost in the slits. Due to space- and the accessibility-constraints, a big gas cell like the one used in SLOWRI is not possible, so we have to use compact cells with a simpler mechanism. The proposed setup is shown in Figure 2. We will place one or more compact gas cells on the side of the main beam path. The gas cell will be filled with 1 bar Ar gas whereby most injected ions can be neutralized. A degrader located in front of the cell will adjust the beam energy to efficiently stop the ions inside the cell. The neutralized atoms will be transported by gas flow toward the exit of the cell, where the atoms can be re-ionized by laser irradiation. This uses the same principle as the conventional IGISOL [5] for the transport and LISOL [6] for the re-ionization scheme.

Although the size of the cell is limited, the high-pressure Ar will enable efficient stopping of energetic RI-beams. In order to ionize the neutralized RI-atoms efficiently, a multi-step resonance ionization method will be used with high power and high repetition rate pulsed lasers. Resonance ionization within a gas cell has been studied intensively by Leuven [6], Mainz [7], and Jyväskylä [8] groups. The re-ionized RI ions from the gas cell will be transported by rf-sextupole ion beam guide (SPIG) [9] through multiple differential pumping stages to an electro-magnetic mass separator. Although the new method is not as universal as the original SLOWRI, the purity of ions can be extremely high. Separations by $A / Z$ are made at BigRIPS, by $Z$ via laser ionization, and by $A$ at the mass separator.

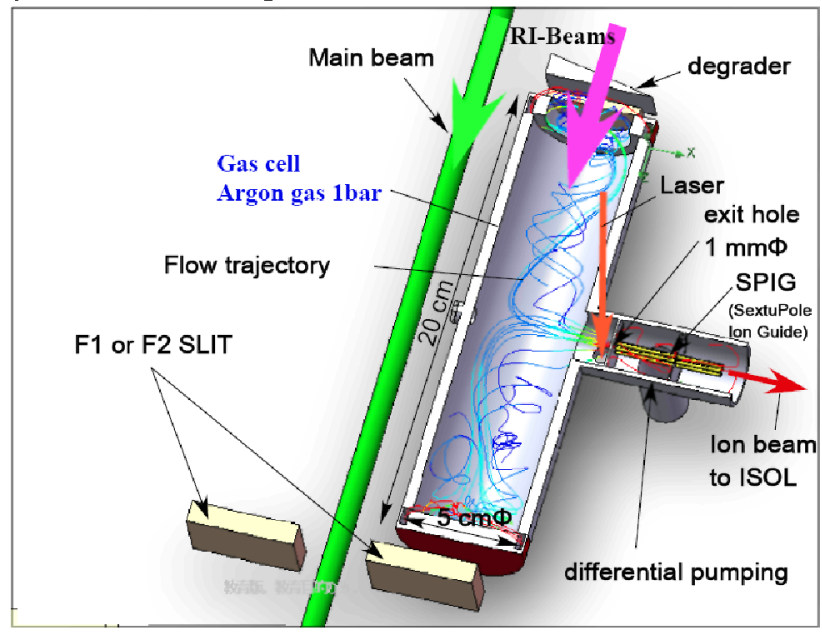

FIGURE 2. Schematic image of the laser ionization gas catcher setup. While the main beam is not disturbed, the contaminant beams are stopped in the Ar gas cell and the neutralized atoms are transported by gas flow towards the exit and then, re-ionized by resonance-laser irradiation.

\section{SOME EXPECTED FEATURES}

Here we discuss some expected advantages of the new method in terms of the problems found in the development of the other gas catcher systems. 


\section{Stopping capability}

In order to stop the high-energy RI-beams from a projectile fragmentation reaction, a large stopping capability of more than $10 \mathrm{MeV} /$ nucleon is required. In contrast to conventional gas catcher systems where the ions survive in the gas cell due to high ionization potential of $\mathrm{He}$, the proposed cell has no reason to use He since the ions must be neutralized. When Ar gas is used, the stopping capability is simply enhanced by a factor of 10 compared to He with same gas cell size. A gas cell filled with 1 bar Ar with a length of $20 \mathrm{~cm}$ can stop ions of less than $13 \mathrm{MeV} /$ nucleon for heavier nuclei of $A>50$ according to Fig. 3 . This implies that a wide acceptance of high energy RI-beams with an appropriate degrader thickness is expected even with a small gas cell. The stopping efficiency for a realistic case was calculated: a $300-\mathrm{MeV} /$ nucleon, $A=78$ nuclei with a momentum spread of $+-3 \%$, an emittance of $+-5 \mathrm{~mm} \mathrm{x}+-40 \mathrm{mrad}$ and a momentum dispersion of $20 \mathrm{~mm} / \%$, can be as high as $40 \%$ and $20 \%$ for a $20 \mathrm{~cm}$ cell and a $5 \mathrm{~cm}$ cell, respectively. In this efficiency, the dominant loss is attributed to those ions which are not transported to the cell due to the large momentum spread and the limited size of the cell.

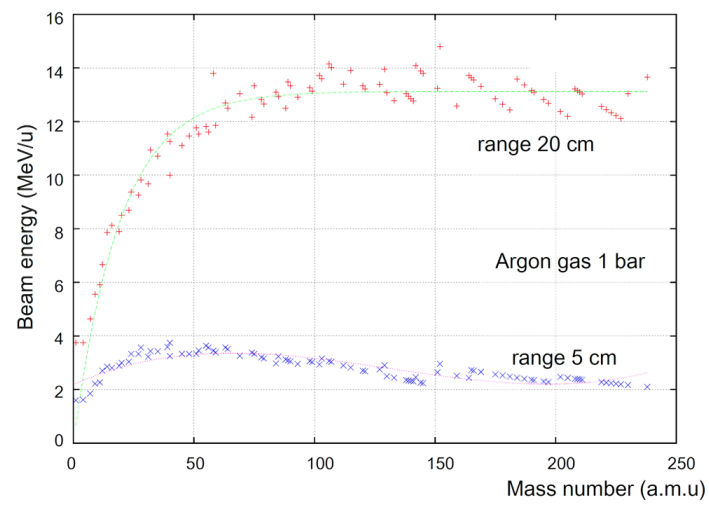

FIGURE 3. Stopping capability of an Ar gas cell ( 1 bar) with a thickness of $20 \mathrm{~cm}$ and $5 \mathrm{~cm}$ plotted as a function of ions mass. The calculation is performed by SRIM2008.

\section{Accessibility of short-lived nuclides}

One of the important issues in the gas catcher system is the extraction time to avoid the decay loss of short-lived isotopes. The proposed gas cell solely uses a gas flow to guide stopped nuclei towards the exit hole of the cell. This extraction time is determined by the volume of the cell and the conductance of the exit hole. The accessibility for short-lived nuclides was evaluated by using a flow calculation and a macroscopic simulation including a diffusion loss. The diffusion loss is estimated to be about $35 \%$ for proposed geometry. Two types of cell, $20 \mathrm{~cm} \times 5 \mathrm{~cm} \Phi$ and $5 \mathrm{~cm}$ x 5 $\mathrm{cm} \Phi$ are examined for the flow calculation. In the macroscopic simulation, the initial positions of the stopped ions are assumed to be distributed uniformly in the cell. The local survival probability of short-lived nuclei to reach the exit from a given position in the cell before decaying can be evaluated from the half-life and the extraction time. The total survival efficiency was then evaluated by integrating the local survival 
efficiency over the entire volume of the cell. The evaluated total efficiency shown in Fig. 4 is obtained from a product of the total survival efficiency, the stopping efficiency and an assumed additional efficiency of $10 \%$. This additional efficiency consists of the laser ionization efficiency and other possible loss, such as molecular formation, beam transport to SLOWRI. For atoms at ground state come out through the exit of the cell can be ionized with a $100 \%$ efficiency, however, these atoms in meta-stable states or in molecular forms cannot be ionized. From the experimental result at Leuven for $\mathrm{Ni}$ isotope [12], this additional efficiency is evaluated to be $10 \%$. The evaluated total efficiency suggests that more than $1 \%$ of overall efficiency is still expected for nuclei with a half-life of longer than $1 \mathrm{~s}$.

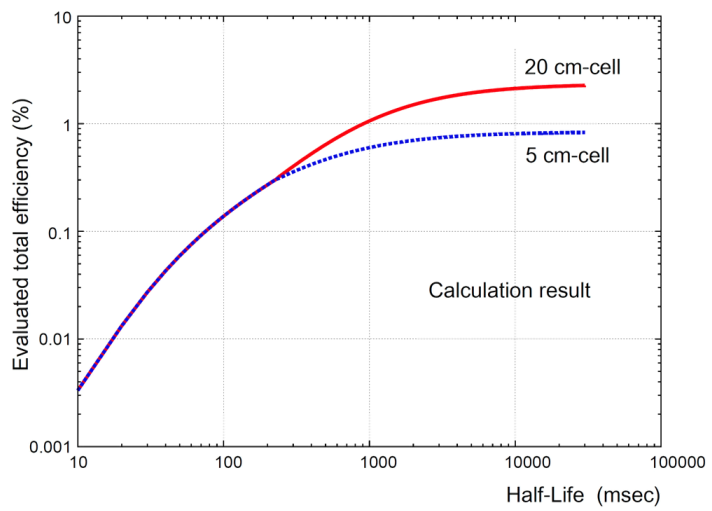

FIGURE 4. Evaluated total efficiency for incoming RI-ions extracted from the cell before decaying. The beam is assumed to be a $300-\mathrm{MeV} /$ nucleon, $A=78$ ion-beam with an emittance of $+-5 \mathrm{~mm} \mathrm{x}+-$ $40 \mathrm{mrad}$, a momentum spread of $3 \%$, and a momentum dispersion of $20 \mathrm{~mm} / \%$ at an wedge-shaped degrader.

\section{Impurity}

The purity of the beams from the laser ionization gas cell is the highest compared with the other schemes. However, impurities in the buffer gas may cause problems such as to induce a molecular formation. In general, re-ionization of molecular beam is not simple. Different from the other types of the gas cell, there is no need to install insulators or electrodes in the proposed gas cell. So that the cell can be made from a UHV grade metallic chamber. Thus the purity of the Ar buffer gas can be maintained very high.

\section{Space charge}

Historically some distinct phenomena have been observed in the various gas catcher systems. The most serious problem is that the extraction efficiency from the cell diminishes with increasing rates of incoming particles $[10,11]$. This phenomenon can be explained as an effect of space charge in the cell. During the process of stopping in the gas cell, one incoming ion produces more than $10^{5}$ ion-electron pairs and the electrons are removed quickly by the applied dc field, while the buffer gas ions remain in the cell for a longer period of time. The buffer gas ions distort the 
external field which is applied to transport the thermal ions towards the exit. The distorted electric field causes losses in the transport of RI-ions. The equilibrium spacecharge density is proportional to the square-root of the beam intensity and the transport efficiency is roughly proportional to the reciprocal of the charge density [11]. In the proposed scheme, however, the RI-ions are intentionally neutralized and transported exclusively by the gas flow. The effect of the space-charge must be minimal. A drastic efficiency enhancement for high-intensity beam was observed using a laser ionization gas cell at LISOL Leuven [12] (Fig. 5). A high efficiency is still preserved with $10^{10} \mathrm{pps}$ beam of ${ }^{58} \mathrm{Ni}$.

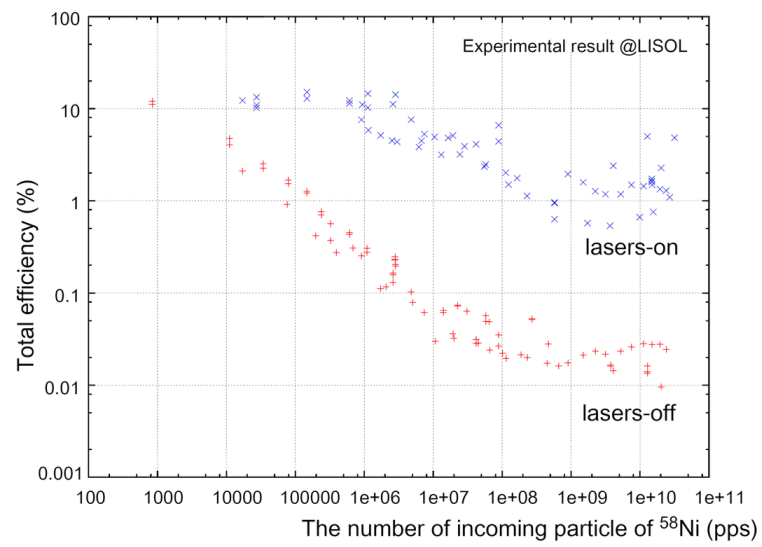

FIGURE 5. Experimentally measured total efficiency of the laser ionization gas cell for ${ }^{58} \mathrm{Ni}$ at Leuven [12]. The total efficiency is defined as the ratio of the number of mass separated ${ }^{58} \mathrm{Ni}$ ions extracted from the gas cell to the number of ${ }^{58} \mathrm{Ni}$ ions entering the cell. More than $1 \%$ efficiency was obtained for high-intensity beams of $>10^{10} \mathrm{pps}$.

\section{SUMMARY}

We have presented a new experimental method to provide parasitic slow RI-beams from a projectile fragment separator without disturbing the primary RI-beam experiment. This is an additional option for RIKEN's universal slow RI-beam facility, SLOWRI.

\section{REFERENCES}

1. T. Kubo, Nucl. Instr. Meth. B204 (2003) 97.

2. M. Wada et al., Nucl. Instr. Meth. B 204 (2003) 570

3. T. Nakamura et al., Phys. Rev. A 74 (2006) 052503.

4. K. Okada et al., Phys. Rev. Lett. 101(2008) 212502.

5. J. Äysto, Nucl. Phys. A693 (2001) 477.

6. Yu. Kudryavtsev et al., Nucl. Phys. A701 (2002) 465.

7. K. Blaum et al. Nucl. Inst. Meth. B204 (2003) 331.

8. I.D. Moore et al., J. Phys. G31 (2005) s1499.

9. H. J. Xu, et al.,Nucl. Inst. Meth. A333 (1993) 274.

10. K. Morita et al., Nucl. Inst. Meth. B26 (1987) 406.

11. A. Takamine et al., Rev. Sci. Inst. $76(2005) 103503$.

12. M. Facina et al., Nucl. Inst. Meth. B226 (2004) 401. 